\title{
SVN \\ Hyperdense artery sign, symptomatic infarct swelling and effect of alteplase in acute ischaemic stroke
}

To cite: Wu S, Mair G, Cohen G, et al. Hyperdense artery sign, symptomatic infarct swelling and effect of alteplase in acute ischaemic stroke. Stroke \& Vascular Neurology 2021;6: e000569. doi:10.1136/svn2020-000569

- Additional material is published online only. To view please visit the journal online (http://dx.doi.org/10.1136/svn2020-000569).

Received 10 August 2020 Revised 10 September 2020 Accepted 7 October 2020 Published Online First 27 November 2020
Check for updates

(C) Author(s) (or their employer(s)) 2021. Re-use permitted under CC BY. Published by BMJ.

For numbered affiliations see end of article.

Correspondence to Dr Ming Liu;

wyplmh@hotmail.com

Dr Joanna M Wardlaw; joanna.wardlaw@ed.ac.uk

Simiao Wu (10 , ${ }^{1,2}$ Grant Mair (1) , ${ }^{2}$ Geoff Cohen, ${ }^{2}$ Zoe Morris, ${ }^{2}$ Anders von Heijne, ${ }^{3}$ Nick Bradey, ${ }^{4}$ Lesley Cala, ${ }^{5}$ Andre Peeters (D) , ${ }^{6}$ Andrew J Farrall, ${ }^{2}$ Alessandro Adami (D) , ${ }^{7}$ Gillian Potter, ${ }^{8}$ Ming Liu, ${ }^{1}$ Richard I Lindley, ${ }^{9,10}$ Peter A G Sandercock (D) ,2 Joanna M Wardlaw (D), 2,11 On behalf of the IST-3 Collaborative Group

\section{ABSTRACT}

Background Alteplase improves functional outcomes of patients with acute ischaemic stroke, but its effects on symptomatic infarct swelling, an adverse complication of stroke and the influence of CT hyperdense artery sign (HAS) are unclear. This substudy of the Third International Stroke Trial aimed to investigate the association between HAS and symptomatic infarct swelling and effect of intravenous alteplase on this association.

Methods We included stroke patients whose prerandomisation scan was non-contrast CT. Raters, masked to clinical information, assessed baseline (prerandomisation) and follow-up (24-48 hours postrandomisation) CT scans for HAS, defined as an intracranial artery appearing denser than contralateral arteries. Symptomatic infarct swelling was defined as clinically significant neurological deterioration $\leq 7$ days after stroke with radiological evidence of midline shift, effacement of basal cisterns or uncal herniation.

Results Among 2961 patients, HAS presence at baseline was associated with higher risk of symptomatic infarct swelling (OR 2.21; $95 \% \mathrm{Cl} 1.42$ to 3.44). Alteplase increased the risk of swelling (OR 1.69; $95 \% \mathrm{Cl} 1.11$ to 2.57), with no difference between patients with and those without baseline HAS $(p=0.49)$. In patients with baseline HAS, alteplase reduced the proportion with HAS at follow-up (OR 0.67; $95 \% \mathrm{Cl} 0.50$ to 0.91 ), where HAS disappearance was associated with reduced risk of swelling (OR $0.25,95 \% \mathrm{Cl} 0.14$ to 0.47 ).

Conclusion Although alteplase was associated with increased risk of symptomatic infarct swelling in patients with or without baseline HAS, it was also associated with accelerated clearance of HAS, which in return reduced swelling, providing further mechanistic insights to underpin the benefits of alteplase.

\section{INTRODUCTION}

Symptomatic infarct swelling is a devastating neurological complication of acute ischaemic stroke. ${ }^{1}$ Currently, there is insufficient evidence to inform its prevention and management in practice, ${ }^{2}$ although observational studies have shown that persistent arterial occlusion was associated with more infarct swelling and reperfusion appeared to reduce the risk of swelling. ${ }^{3}$ The hyperdense artery sign (HAS) on non-contrast CT is a widely accessible imaging sign in stroke practice, ${ }^{45}$ which is highly specific and moderately sensitive for detection of intracranial arterial obstruction by thrombus. ${ }^{6}$ However, the relationship between HAS and swelling is less clear as divergent findings were reported in previous studies. ${ }^{7-12}$

Although there are concerns that reperfusion therapies might increase infarct swelling, the Multicentre Randomised Clinical Trial of Endovascular Treatment for Acute Ischaemic Stroke in the Netherlands (MR CLEAN) showed that successful reperfusion by mechanical clot retrieval reduced the development of midline shift. ${ }^{13}$ Nevertheless, the impact of arterial recanalisation by intravenous thrombolysis on the development of severe infarct swelling is unknown. ${ }^{14}$ In the Third International Stroke Trial (IST-3), intravenous alteplase increased HAS clearance, and the disappearance of HAS was associated with better functional outcome. ${ }^{15}$ Therefore, intravenous thrombolysis is a promising therapy for patients with HAS, particularly where thrombectomy is not available.

In this study, we used data from IST-3 to investigate whether the presence of HAS on baseline CT predicted development of symptomatic infarct swelling and the effect of intravenous alteplase on this association.

\section{METHODS}

\section{Third International Stroke Trial}

IST-3 was an international, multicentre, prospective, randomised, open- blinded endpoint trial in acute ischaemic stroke comparing intravenous alteplase versus control, recruiting between May 2000 and July 2011. IST-3 is registered, ISRCTN25765518. The detailed study design and methods of the 
IST-3 have been described elsewhere. ${ }^{16-19}$ Briefly, patients were eligible if they had clinically definite acute stroke of any severity (assessed prior to randomisation with the National Institute of Health Stroke Scale (NIHSS)), age $\geq 18$ years, for whom CT or MRI had excluded intracranial haemorrhage and any structural stroke mimics, and the treatment could be started within 6 hours of stroke onset, without clear indications for, or contraindications to, intravenous thrombolysis with alteplase. In total, 3035 patients were randomised to receive standard care plus intravenous alteplase $(0.9 \mathrm{mg} / \mathrm{kg})$ or standard care alone. No intra-arterial therapy was used. Primary outcome was the functional status at 6 months assessed with the Oxford Handicap Scale. Results were analysed on an intention-totreat basis. Collaborators and funders of IST-3 are listed in the online supplemental material 1.

\section{Imaging protocol and analysis}

The IST-3 imaging protocol has been described previously. ${ }^{1620}$ All patients had a brain CT or MRI scan before randomisation and a follow-up scan at 24-48 hours after randomisation. A repeat brain scan was required if the patient deteriorated neurologically or intracranial haemorrhage was suspected for any reason. Non-contrast CT scans were required to cover the brain from foramen magnum to vertex, with maximum slice thickness of $4-5 \mathrm{~mm}$ through the posterior fossa and $8-10 \mathrm{~mm}$ for the cerebral hemispheres, with no slice gap. Scans were windowed on 80 Hounsfield Units (HU) width and centre level of 35-40 HU. A centralised panel of neurologists and neuroradiologists experienced in acute stroke imaging evaluated all relevant CT scans using a validated, prespecified rating proforma (www.ed.ac.uk/clinicalsciences/edinburgh-imaging/research/analysis-andprocessing/image-analysis-tools-downloads/all-the-edinburgh-imaging-rating-tools). ${ }^{51621}$ All raters were blinded to clinical data and imaging at other time points.

HAS presence was determined visually on non-contrast CT on both baseline (prerandomisation) and follow-up (24-48 hour postrandomisation) scans. No objective HU measurements were made, as in prior research, we found that this approach did not materially increase sensitivity or specificity for acute thrombus. ${ }^{22}{ }^{23}$ The raters identified HAS by deciding if an intracranial artery appeared denser than adjacent arteries or than equivalent contralateral arteries, but not due to calcification, at the following locations: internal carotid artery, mainstem or sylvian branches of middle cerebral artery (MCA), anterior cerebral artery (ACA), posterior cerebral artery (PCA), vertebral or basilar arteries. In a large observer reliability study based on the IST-3 imaging data, the inter-rater and intrarater reliability of HAS identification by Krippendorff $\alpha$ was 0.59 and 0.58 , respectively. ${ }^{6}$ The status of HAS was recorded as 'persistent' for HAS present on both baseline and follow-up CT; 'disappeared' for HAS present at baseline but not follow-up; 'newly developed' for HAS not present on baseline CT but on follow-up CT' and 'none' for HAS absent at both baseline and follow-up.
We assessed the extent of the acute ischaemic lesion using the IST-3 score, which includes all arterial territories and differentiates infarct extent from brain swelling. ${ }^{16}$ The score has been widely validated and shown to have better inter-rater reliability and dynamic range than other infarct extent scores. ${ }^{162124}$ We condensed the full-lesion extent scores into four groups for analysis, as previously ${ }^{16}$ : small infarcts, defined as lacunar, small cortical, small cerebellar, less than half of brainstem or less than half of the ACA or PCA territory; medium infarcts, defined as striatocapsular, the anterior or posterior half of the peripheral MCA territory or more than half of the ACA or PCA territory; large infarcts, defined as the whole of the peripheral MCA territory or all the MCA territory and very large infarcts, defined as the whole MCA and PCA territory, the whole MCA and ACA territory or all three territories.

\section{Definition of symptomatic infarct swelling}

The outcome in this analysis was prespecified in the IST-3 study protocol, ${ }^{17}{ }^{19}$ where symptomatic infarct swelling was defined as clinically significant neurological deterioration (as assessed and reported by trial investigators according to their local protocol) or death $\leq 7$ days of randomisation, accompanied by evidence of significant infarct swelling as determined by the independent masked expert assessment of the scan. Significant infarct swelling was defined as any degree of the shift of the midline cerebral structures away from their usual central location in the brain, effacement of the basal cisterns or uncal herniation on a follow-up scan or finding severe infarct swelling at autopsy if not rescanned before death. The presence of some degree of haemorrhagic transformation was permitted, provided it was not considered by the expert imaging rater to be a major contributor to the mass effect.

\section{Statistical analysis}

Patients whose prerandomisation radiological assessment was a non-contrast CT scan that had undergone blinded central review were included in this analysis. We excluded the few patients with prerandomisation MRI scans. We compared clinical characteristics between patients with and without symptomatic infarct swelling, using t-tests, Wilcoxon test or $\chi^{2}$ tests as appropriate. We performed tests of interaction to compare ORs for the association between HAS and symptomatic infarct swelling in patients with an initial NIHSS $<15$ versus $\geq 15$; patients with large to very large infarcts versus small to medium infarcts and patients allocated to alteplase versus those on control. We also used logistic regression with symptomatic infarct swelling as dependent variable to calculate ORs for HAS, adjusted for the effects of patient age, NIHSS, extent of ischaemic lesion and allocation to alteplase. IST-3 was powered to detect the clinical difference in the primary outcome, and the clinical (symptomatic infarct swelling) and imaging (HAS) variables for secondary analysis were prespecified. ${ }^{17} 1820$ In addition, we had been cautious 
with the number of variables allowed in regression analysis to satisfy the sample size criterion of $\geq 10$ events per variable. $^{25} 26$ Therefore, the study was anticipated to be sufficient to enable the assessment of the effects of alteplase on HAS and on infarct swelling. All analyses were performed with SAS software V.9.4 (SAS Institute, Cary, North Carolina, USA). All tests were two sided with $\mathrm{p}<0.05$ as significant.

\section{RESULTS}

Of 3035 patients randomised in the IST-3, non-contrast CT scans were centrally reviewed for 2961 patients at baseline (18/3035 patients with baseline CT were not available for central review and MRI only was performed at baseline in the remaining 56/3035 patients) and for 2731 patients at both baseline and follow-up. HAS data were missing for one follow-up CT due to poor quality scan, thus 2730 patients had HAS data at both baseline and follow-up.

\section{Associations between baseline HAS and symptomatic infarct swelling}

Of 2961 patients with baseline CT scans (mean age, 77.4 (SD, 12.1) years; men, $48.2 \%$; onset to scan time, median 3 (IQR, 2-4hours), 716 (24.2\%)) demonstrated baseline HAS and $107(3.6 \%)$ developed symptomatic infarct swelling $\leq 7$ days after stroke onset (median, 3 (IQR 1-4) days). Patients with baseline HAS had higher NIHSS (median, 16 (IQR 10-20]) than those without HAS (median, 9 (IQR 6-16); p<0.001). Patients were more likely to develop symptomatic infarct swelling if they had baseline HAS (unadjusted OR, 4.83 (95\% CI 3.25 to 7.16); $\mathrm{p}<0.001$ ), NIHSS $\geq 15$ (unadjusted OR, 8.82 (95\% CI 5.34 to 14.56$) ; \mathrm{p}<0.001$ ), large to very large infarcts (unadjusted OR, 5.76 (95\% CI 3.89 to 8.52 ); $\mathrm{p}<0.001$ ) and were allocated to alteplase (unadjusted OR, 1.70 (95\% CI 1.14 to 2.53 ); $\mathrm{p}=0.008$ ). There was no association between symptomatic infarct swelling and age, sex or time from onset to randomisation (table 1). The sensitivity and specificity of baseline HAS for predicting symptomatic infarct swelling was 0.59 (95\% CI 0.50 to 0.64 ) and 0.77 (95\% CI 0.73 to 0.82 ), respectively. Baseline HAS was associated with a higher risk of death $\leq 7$ days (unadjusted OR, 2.16 (95\% CI 1.66 to 2.82); $\mathrm{p}<0.001$ ), and the development of symptomatic infarct swelling did not change the adverse association of baseline HAS with early death ( $p$ for interaction 0.11).

In multivariable analysis, the risk of symptomatic infarct swelling increased independently with higher NIHSS, larger infarcts, presence of baseline HAS and allocation to alteplase and decreased with increasing age (table 2). The association between HAS and symptomatic infarct

Table 1 Baseline characteristics and 7 day fatality of patients with and without symptomatic infarct swelling

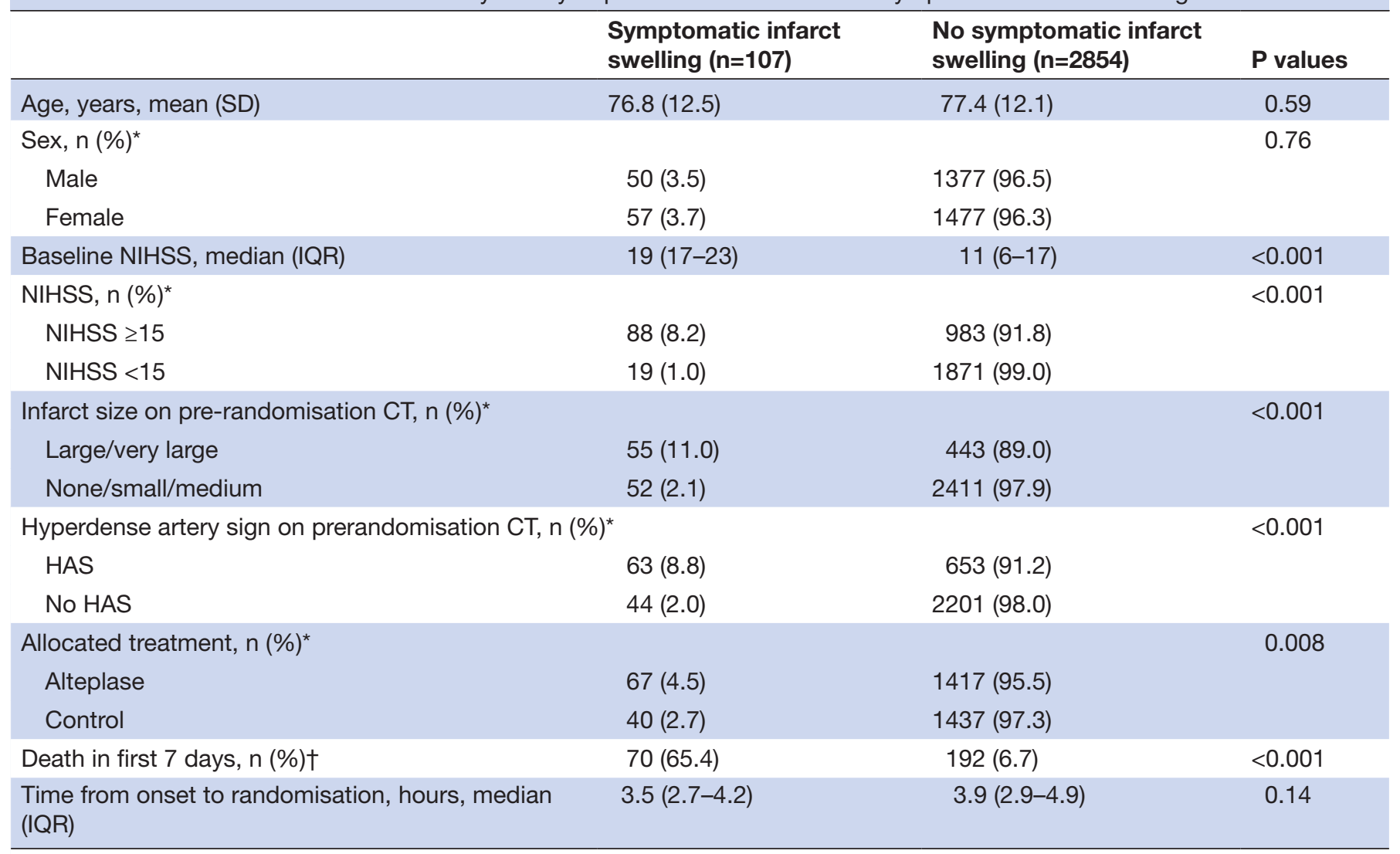

*The proportion of patients with/without infarct swelling in each row characteristic subgroup.

†The proportion of patients who died $\leq 7$ days in patients with/without symptomatic infarct swelling.

HAS, hyperdense artery sign; NIHSS, National Institute of Health Stroke Scale. 
Table 2 Multivariable logistic regression for symptomatic infarct swelling

\begin{tabular}{lrrrc}
\hline Factors & Beta & OR & 95\% CI & P values \\
\hline Age $^{*}$ & -0.02 & 0.98 & 0.97 to 0.99 & 0.04 \\
NIHSS $^{*}$ & 0.14 & 1.15 & 1.11 to 1.18 & $<0.001$ \\
Large to very large infarct (vs small to medium infarct) & 0.89 & 2.44 & 1.57 to 3.79 & $<0.001$ \\
Allocated alteplase (vs control) & 0.53 & 1.69 & 1.11 to 2.57 & 0.01 \\
HAS presence (vs absence) & 0.79 & 2.21 & 1.42 to 3.44 & $<0.001$ \\
\hline
\end{tabular}

*Entered as linear variables, where the OR reflected the expected change in odds for one-point increase in NIHSS or 1-year increase in age. HAS, hyperdense artery sign; NIHSS, National Institute of Health Stroke Scale.

swelling was stronger among patients with baseline NIHSS $<15$ than those with NIHSS $\geq 15$ (OR, $7.44(95 \%$ CI 2.97 to 18.64 ) vs OR, 2.49 (95\% CI 1.60 to 3.88); $p$ for interaction 0.04), but this interaction between HAS and NIHSS was no longer present when a linear function of NIHSS was assessed in multivariable regression, adjusted for age, baseline HAS, large to very large infarct and allocation to alteplase $(\mathrm{p}=0.37$, data not shown $)$. The effects of alteplase ( $p$ for interaction 0.49 ) and of large to very large infarcts ( $\mathrm{p}$ for interaction 0.81) on increased risk of symptomatic infarct swelling showed no significant interaction with baseline HAS presence or absence.

\section{Effect of alteplase on the development of symptomatic infarct swelling}

Of 2730 patients with information on HAS on both baseline and follow-up CT, HAS was identified on 673 (24.7\%) baseline scans, of whom 60 (8.9\% of 673) patients developed symptomatic infarct swelling. Alteplase increased the risk of symptomatic infarct swelling in general, and this pattern persisted regardless of changes in HAS (table 3 ).

Of 673 patients with baseline HAS, alteplase reduced persistent HAS (152/350, 43.4\%, allocated alteplase vs $172 / 323,53.3 \%$, allocated control; unadjusted OR, 0.67 (95\% CI 0.50 to 0.91$) ; \mathrm{p}=0.01)$. Patients with higher NIHSS (median, 17 (IQR 13-21) vs median, 14 (IQR $9-19) ; \mathrm{p}<0.001)$ and large to very large infarcts $(152 / 272$, $55.9 \%$ vs $172 / 401,42.9 \%$; $\mathrm{p}<0.001$ ) were more likely to have persistent HAS. Patients whose HAS disappeared were less likely to develop symptomatic infarct swelling than those with persistent HAS on follow-up (14/349, $4.0 \%$, disappeared HAS vs $46 / 324,14.2 \%$, persistent HAS; unadjusted OR, 0.25 (95\% CI 0.14 to 0.47 ); $\mathrm{p}<0.001$ ).

Of 2057 patients without baseline HAS, there were fewer newly developed HAS in the alteplase group $(90 / 1047,8.6 \%)$ than the control group (106/1010, $10.5 \%)$, although the difference was not statistically significant (unadjusted OR, 0.80 (95\% CI 0.60 to 1.09); $\mathrm{p}=0.16)$. Patients with newly developed HAS $(\mathrm{n}=196)$ on follow-up CT were more likely to develop infarct swelling than the remaining 1861 patients (unadjusted OR, 4.19 (95\% CI 2.16 to 8.16$) ; \mathrm{p}<0.001)$.

\section{DISCUSSION}

In this IST-3 cohort of 2961 patients with baseline CT, the presence of HAS, either on baseline CT, or persisting or newly developed on follow-up CT, was associated with a higher risk of developing symptomatic infarct swelling $\leq 7$ days after stroke. Alteplase increased the risk of developing infarct swelling at follow-up whether or not HAS was present at baseline. However, at follow-up, alteplase promoted HAS disappearance in patients with baseline HAS and reduced development of new HAS in those without baseline HAS, and these patients then had less risk of infarct swelling. These findings provide further evidence of benefits of alteplase in not only removing thrombus but also reducing secondary

Table 3 Effect of alteplase on the development of symptomatic infarct swelling by change in HAS between baseline and follow-up

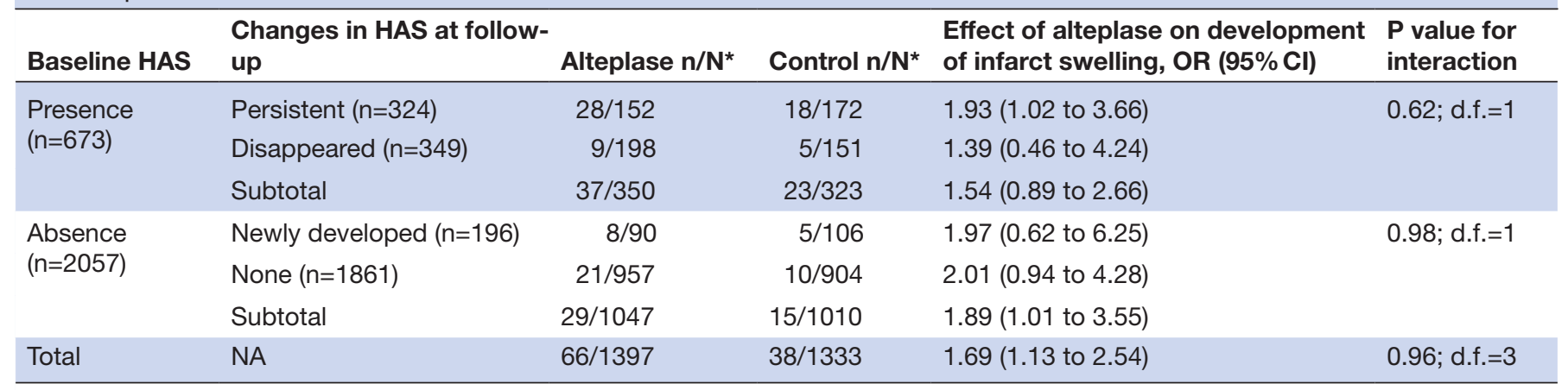

${ }^{*} \mathrm{~N}$ or $\mathrm{n}=$ number of patients in each subgroup of morphological changes in HAS presence, $\mathrm{n} / \mathrm{N}$ : $\mathrm{n}=$ number of patients with infarct swelling.

d.f., degree of freedom; HAS, hyperdense artery sign; NA, not applicable. 
adverse effects of stroke, thereby altering the natural history of ischaemic lesion progression towards a better chance of recovery. Alteplase became a World Health Organisation Essential Medicine in 2018. It is widely used over the world in patients with acute ischaemic stroke and is available for patients who do not meet criteria for thrombectomy, where thrombectomy is unavailable, and as adjunct therapy to thrombectomy. Therefore, these results are relevant to a large proportion of patients with acute ischaemic stroke.

Previous studies reported that HAS at baseline was associated with infarct swelling in patients with stroke treated with alteplase. ${ }^{27}$ We have clarified that HAS increases symptomatic infarct swelling in both patients allocated alteplase and those allocated control, and separately, that alteplase increases symptomatic infarct swelling regardless of the presence or absence of baseline HAS. In this large cohort, baseline HAS showed moderate sensitivity and specificity for development of symptomatic infarct swelling, indicating that HAS is just one of several predictors that contributes to swelling. The presence of HAS is associated with more severe stroke and larger infarct size ${ }^{29}{ }^{30}$ where the latter two also predict symptomatic infarct swelling. ${ }^{331}$ We further show that HAS increases symptomatic infarct swelling independent of NIHSS and infarct size. Our results also suggest that HAS in patients with NIHSS $<15$ may be a useful warning of increased risk of symptomatic infarct swelling, that is, in patients who may otherwise not be expected to develop this complication, but this requires further testing. In addition, we confirm that older patients are less likely to develop symptomatic infarct swelling independent of HAS, NIHSS, large infarct size and alteplase, possibly attributable to the buffering space for swelling provided by the age-related brain atrophy.

Although alteplase promoted HAS disappearance and improved functional outcome,,${ }^{17}$ we show that it increases symptomatic infarct swelling in general, independent of HAS presence. However, in patients with baseline HAS, who therefore have a high risk of symptomatic infarct swelling, alteplase promoted HAS disappearance and thus reduced symptomatic infarct swelling. Therefore, it appears that the increased risk of symptomatic infarct swelling by alteplase was outweighed by the benefit that resulted from its ability to accelerate racanalisation in this patient group with baseline HAS. Despite this benefit, some patients with baseline HAS allocated to alteplase had the persistent risk of swelling, which may be partly due to unsuccessful reperfusion. Similar findings were reported in the MR CLEAN trial, where successful recanalisation but not the allocation to endovascular treatment was associated with a lower risk of midline shift on follow-up scans. ${ }^{13}$ Although thrombectomy may provide a higher proportion of recanalisation and some patients in IST-3 described in the present analysis would now be candidates for thrombectomy, intravenous alteplase is more accessible than thrombectomy for stroke patients in many areas and should not be withheld from patients presenting with HAS even where thrombectomy is being considered. $^{32}$ Our findings from the IST-3, together with the benefit shown in the MR CLEAN trial, provide evidence that patients at risk of infarct swelling could benefit from reperfusion therapies, either by intravenous thrombolysis or mechanical clot retrieval.

Our study has some limitations. First, we assessed HAS qualitatively on non-contrast CT without HU measurement, since the visual assessment of HAS is clinically relevant and highly specific for angiographic obstruction with good reliability across a wide range of raters, ${ }^{4-6}$ and that HU measurement does not improve sensitivity or specificity. ${ }^{22}{ }^{23}$ Second, we did not verify the association between HAS and angiographic arterial obstruction for current analysis, because we do not have angiography for all of these patients and notwithstanding, we wish to focus on the imaging sign on non-contrast CT-a technique that is widely accessible and rapidly translatable in clinical practice, and since many patients, in many parts of the world, for many reasons, do not receive routine CT angiography. Therefore, our findings could translate immediately to the generality of stroke worldwide.

\section{CONCLUSION}

Patients with HAS on non-contrast CT $\leq 48$ hours after stroke onset were more likely to develop symptomatic infarct swelling within 7 days after stroke. Overall, intravenous alteplase increased the risk of symptomatic infarct swelling, but this risk reduced in patients whose baseline HAS had disappeared at follow-up, likely mediated by removal of obstructing thrombus and improved tissue reperfusion, consistent with the clinical benefit of intravenous alteplase.

\section{Author affiliations}

${ }^{1}$ Department of Neurology, West China Hospital, Sichuan University, Chengdu, Sichuan, China

${ }^{2}$ Centre for Clinical Brain Sciences, The University of Edinburgh, Edinburgh, UK ${ }^{3}$ Department of Clinical Sciences, Danderyd Hospital, Stockholm, Sweden ${ }^{4}$ Neuroradiology, James Cook University Hospital, South Tees Hospital NHS Trust, Middlesbrough, UK

${ }^{5}$ Division of Pathology and Laboratory Medicine, School of Medicine, The University of Western Australia, Perth, Western Australia, Australia

${ }^{6}$ Department of Neurology, Cliniques universitaires Saint-Luc, Bruxelles, Belgium ${ }^{7}$ Stroke Center, IRCCS Sacro Cuore Don Calabria, Verona, Italy

${ }^{8}$ Department of Neuroradiology, Manchester Centre for Clinical Neurosciences, Salford Royal NHS Foundation Trust, Salford, UK

${ }^{9}$ Westmead Applied Research Centre, Westmead Clinical School, The University of Sydney, Sydney, New South Wales, Australia

${ }^{10}$ The George Institute for Global Health, Newtown, New South Wales, Australia ${ }^{11}$ UK Dementia Research Institute Centre at the University of Edinburgh, Edinburgh, United Kingdom

Funding This study was primarily funded by the UK Medical Research Council (MRC G0400069 and EME-09-800-15). Other fundings are detailed in the Supplemental Material. SW acknowledges funding from the National Natural Science Foundation of China (81701156). GM acknowledges funding from the Stroke Association (SA L-SMP 18\1000). ML acknowledges funding from the Key Research and Development Program, Science \& Technology Department of Sichuan Province (2017SZ0007), the Major International (Regional) Joint Research Project, National Natural Science Foundation of China (81620108009), the National Key Research and Development Program of China, Ministry of Science and Technology of China (2016YFC1300500-505), and the 1.3.5 Project for Disciplines of Excellence, West China Hospital, Sichuan University (ZYGD18009). JMW acknowledges funding from the UK Dementia Research Institute (DRI Ltd: Medical Research Council, Alzheimer's Society and Alzheimer's Research UK), British Heart 
Foundation Centre for Research Excellence Award III (RE/18/5/34216) and the Fondation Leducq (16 CVD 05).

Competing interests RIL reports numerous grants during the conduct of the study as detailed in the supplemental material. PAGS reports non-financial support from Boehringer ingelheim, grants from Medical Research Council UK, during the conduct of the study. JMW reports grants from Medical Research Council, Stroke Association, The Health Foundation, Scottish Funding Council, Chief Scientist Office, and Chest, Heart \& Stroke Scotland, during the conduct of the study; grants from British Heart Foundation (Edinburgh Centre for Research Excellence and LACI-2 trial), MRC UK Dementia Research Institute, Fondation Leducq (ref 16 CVD 05), EU Horizon 2020 (PHC-03-15, project No 666881), and 'SVDs@Target', outside the submitted work

Patient consent for publication Not required.

Ethics approval Ethical approval was granted by Multicentre Research Ethics Committees, Scotland (MREC/99/0/78) and by local ethics committees in other countries. Informed consent was obtained for all patients.

Provenance and peer review Not commissioned; externally peer reviewed. Data availability statement The IST-3 data are deposited at https://doi.org/10. $7488 / \mathrm{ds} / 1350$, available on application to the study investigators.

Supplemental material This content has been supplied by the author(s). It has not been vetted by BMJ Publishing Group Limited (BMJ) and may not have been peer-reviewed. Any opinions or recommendations discussed are solely those of the author(s) and are not endorsed by BMJ. BMJ disclaims all liability and responsibility arising from any reliance placed on the content. Where the content includes any translated material, BMJ does not warrant the accuracy and reliability of the translations (including but not limited to local regulations, clinical guidelines, terminology, drug names and drug dosages), and is not responsible for any error and/or omissions arising from translation and adaptation or otherwise.

Open access This is an open access article distributed in accordance with the Creative Commons Attribution 4.0 Unported (CC BY 4.0) license, which permits others to copy, redistribute, remix, transform and build upon this work for any purpose, provided the original work is properly cited, a link to the licence is given, and indication of whether changes were made. See: https://creativecommons.org/ licenses/by/4.0/.

ORCID iDs

Simiao Wu http://orcid.org/0000-0002-4557-7386

Grant Mair http://orcid.org/0000-0003-2189-443X

Andre Peeters http://orcid.org/0000-0001-9989-4283

Alessandro Adami http://orcid.org/0000-0002-9163-4900

Peter A G Sandercock http://orcid.org/0000-0001-8484-0135

Joanna M Wardlaw http://orcid.org/0000-0002-9812-6642

\section{REFERENCES}

1 Balami JS, Chen R-L, Grunwald IQ, et al. Neurological complications of acute ischaemic stroke. Lancet Neurol 2011;10:357-71.

2 Powers WJ, Rabinstein AA, Ackerson T, et al. Guidelines for the early management of patients with acute ischemic stroke: 2019 update to the 2018 guidelines for the early management of acute ischemic stroke: a guideline for healthcare professionals from the American heart Association/American stroke association. Stroke 2019;50:e344-418.

3 Wardlaw JM, Dennis MS, Lindley RI, et al. Does early reperfusion of a cerebral infarct influence cerebral infarct swelling in the acute stage or the final clinical outcome? Cerebrovasc Dis 1993;3:86-93.

4 Wardlaw JM, Mielke O. Early signs of brain infarction at CT: observer reliability and outcome after thrombolytic treatment--systematic review. Radiology 2005;235:444-53.

5 Wardlaw JM, Farrall AJ, Perry D, et al. Factors influencing the detection of early CT signs of cerebral ischemia: an Internet-based, International multiobserver study. Stroke 2007;38:1250-6.

6 Mair G, Boyd EV, Chappell FM, et al. Sensitivity and specificity of the hyperdense artery sign for arterial obstruction in acute ischemic stroke. Stroke 2015;46:102-7.

7 Haring HP, Dilitz E, Pallua A, et al. Attenuated corticomedullary contrast: an early cerebral computed tomography sign indicating malignant middle cerebral artery infarction. A case-control study. Stroke 1999;30:1076-82.

8 Kasner SE, Demchuk AM, Berrouschot J, et al. Predictors of fatal brain edema in massive hemispheric ischemic stroke. Stroke 2001;32:2117-23.
9 Krieger DW, Demchuk AM, Kasner SE, et al. Early clinical and radiological predictors of fatal brain swelling in ischemic stroke. Stroke 1999;30:287-92.

10 Kucinski T, Koch C, Grzyska U, et al. The predictive value of early CT and angiography for fatal hemispheric swelling in acute stroke. AJNR Am J Neuroradiol 1998;19:839-46.

11 Ong CJ, Gluckstein J, Laurido-Soto O, et al. Enhanced detection of edema in malignant anterior circulation stroke (edema) score: a risk prediction tool. Stroke 2017;48:1969-72.

12 Muscari A, Faccioli L, Lega MV, et al. Predicting cerebral edema in ischemic stroke patients. Neurol Sci 2019;40:745-52.

13 Kimberly WT, Dutra BG, Boers AMM, et al. Association of reperfusion with brain edema in patients with acute ischemic stroke: a secondary analysis of the Mr clean trial. JAMA Neurol 2018;75:453-61.

14 Wardlaw JM, Murray V, Berge E, et al. Thrombolysis for acute ischaemic stroke. Cochrane Database of Syst Rev 2014:113:CD000213.

15 Mair G, von Kummer R, Morris Z, et al. Effect of alteplase on the CT hyperdense artery sign and outcome after ischemic stroke. Neurology 2016;86:118-25.

16 IST-3 collaborative group. Association between brain imaging signs, early and late outcomes, and response to intravenous alteplase after acute ischaemic stroke in the third International stroke trial (IST-3): secondary analysis of a randomised controlled trial. Lancet Neurol 2015;14:485-96.

17 IST-3 collaborative group, Sandercock P, Wardlaw JM, et al. The benefits and harms of intravenous thrombolysis with recombinant tissue plasminogen activator within $6 \mathrm{~h}$ of acute ischaemic stroke (the third international stroke trial [IST-3]): a randomised controlled trial. Lancet 2012;379:2352-63.

18 Sandercock P, Lindley R, Wardlaw J, et al. Statistical analysis plan for the third International Stroke Trial (IST-3); part of a 'thread' of reports of the trial. Int J Stroke 2012;7:186-7.

19 Sandercock P, Lindley R, Wardlaw J, et al. Third International stroke trial (IST-3) of thrombolysis for acute ischaemic stroke. Trials 2008:9:37.

20 Wardlaw JM, von Kummer R, Carpenter T, et al. Protocol for the perfusion and angiography imaging sub-study of the third International stroke trial (IST-3) of alteplase treatment within six-hours of acute ischemic stroke. Int J Stroke 2015;10:956-68.

21 Wardlaw JM, von Kummer R, Farrall AJ, et al. A large web-based observer reliability study of early ischaemic signs on computed tomography. the acute cerebral CT evaluation of stroke study (access). PLoS One 2010;5:e15757.

22 Ernst M, Romero JM, Buhk JH, et al. Sensitivity of visual and quantitative detection of middle cerebral artery occlusion on non-contrast-enhanced computed tomography. Neuroradiology 2014;56:1063-8.

23 Mair G, von Kummer R, Lindley RI, et al. Effect of x-ray attenuation of arterial obstructions on intravenous thrombolysis and outcome after ischemic stroke. PLoS One 2015:10:e0145683.

24 Wardlaw JM, Sellar R. A simple practical classification of cerebral infarcts on CT and its interobserver reliability. AJNR Am J Neuroradiol 1994:15:1933-9.

25 Harrell FE, Lee KL, Mark DB. Multivariable prognostic models: issues in developing models, evaluating assumptions and adequacy, and measuring and reducing errors. Stat Med 1996;15:361-87.

26 Peduzzi P, Concato J, Kemper E, et al. A simulation study of the number of events per variable in logistic regression analysis. $J$ Clin Epidemiol 1996;49:1373-9.

27 Strbian D, Meretoja A, Putaala J, et al. Cerebral edema in acute ischemic stroke patients treated with intravenous thrombolysis. Int $J$ Stroke 2013:8:529-34.

28 Thorén M, Azevedo E, Dawson J, et al. Predictors for cerebral edema in acute ischemic stroke treated with intravenous thrombolysis. Stroke 2017;48:2464-71.

29 Manelfe C, Larrue V, von Kummer R, et al. Association of hyperdense middle cerebral artery sign with clinical outcome in patients treated with tissue plasminogen activator. Stroke 1999;30:769-72.

30 Berge E, Nakstad PH, Sandset PM, et al. Large middle cerebral artery infarctions and the hyperdense middle cerebral artery sign in patients with atrial fibrillation. Acta Radiol 2001;42:261-8.

31 Wu S, Yuan R, Wang Y, et al. Early prediction of malignant brain edema after ischemic stroke. Stroke 2018;49:2918-27.

32 Powers WJ, Rabinstein AA, Ackerson T, et al. 2018 guidelines for the early management of patients with acute ischemic stroke: a guideline for healthcare professionals from the American heart Association/ American stroke association. Stroke 2018;49:e46-110. 